\title{
A competitive facility location in a closed form supply chain
}

\author{
Mohammad Ali Mohammadi $^{\mathbf{a}^{*}, \text { Hamid Davoudpour }^{\mathrm{a}} \text { and Zahra Motamedi }}{ }^{\mathrm{b}}$
}

${ }^{a}$ Department of Industrial Engineering and Management Systems Amir Kabir University of Technology, Tehran, Iran ${ }^{b}$ Department of Basic Sciences, Payam Noor University Of Tehran, Iran

\begin{tabular}{l}
\hline C H R O N I C L E \\
\hline Article history: \\
Received October 15, 2013 \\
Accepted January 24, 2014 \\
Available online \\
March 62014 \\
\hline Keywords: \\
Capacitated facility location \\
problem \\
CFLP \\
Lagrangian relaxation \\
Green supply chain management
\end{tabular}

\section{A B S T R A C T}

This paper studies capacitated facility location problem by considering green management
perspectives. The proposed study considers reverse logistic problem as an alternative strategy
for facility location in an attempt to take care of environmental characteristics. The resulted
problem is formulated as mixed integer programming and it is classified as an NP-Hard
problem. Therefore, a Lagrangian relaxation methodology is presented to reduce the complexity
of the proposed problem and the solution has been implemented for some instances to examine
the performance of the proposed study.

(c) 2014 Growing Science Ltd. All rights reserved.

\section{Introduction}

During the past few years, there have been increasing interests to determine a facility in competitive environment (Sridharan, 1995; Nauss, 1978; Küçükdeniz et al., 2012; Yang et al., 2012; Manzour-alAjdad et al., 2012; Rahmaniani \& Ghaderi, 2013). Wu et al. (2006) presented an extension of the capacitated facility location problem (CFLP) by considering the general setup cost functions and multiple facilities in one. The setup costs consist of a fixed term, which is site setup cost along with facility setup costs. The facility setup cost functions were in form of non-linear functions of the size of the facility in the same site. They presented two equivalent mixed integer linear programming (MIP) models for the problem and solved them by general MIP solver. They also developed a Lagrangian heuristic algorithm (LHA) to find near optimal solutions for the proposed NP-hard problem. They also considered the performance of the proposed model was examined against various instances. According to Avella et al. (2009), the Capacitated Facility Location Problem (CFLP) determines a set of facilities with capacity constraints to meet the demands of a set of clients at the minimum cost.

\footnotetext{
* Corresponding author.

E-mail addresses: m.ali.mohammadi@aut.ac.ir (M. A. Mohammadi)

C 2014 Growing Science Ltd. All rights reserved. doi: $10.5267 /$ j.dsl.2014.3.004
} 
Avella et al. (2009) proposed a simple and effective heuristic for large-scale instances of CFLP. The heuristic was based on a Lagrangean relaxation applied to select a subset of so called "promising" variables forming the core problem and on a Branch-and-Cut algorithm, which handles the core problem. Jain and Vazirani (2001) presented an approximation algorithm for metric facility location and k-median problems using the primal-dual schema and Lagrangian relaxation. Pirkul and Jayaraman (1998) presented a multi-commodity, multi-plant, capacitated facility location problem and proposed an efficient heuristic solution to solve the resulted problem for some large-scale problems.

\section{The proposed study}

The proposed study considers reverse logistic problem as an alternative strategy for facility location in an attempt to take care of environmental characteristics. The proposed study of this considers two type of customers. The first groups, $J_{1}$, is associated with the customers who are interested in purchasing the new products while the second group, $J_{2}$, is related to customers who are interested in returned or used products. There are two types of facilities with the proposed study of this paper. The first group, $A$, is associated with new rivals while the second group, $B$, is related to existing facilities. All facilities are assumed to be connected and there are two types of forward and return paths. In forward processing facility, product is produced and it is forwarded to customers while collection facility is responsible to collect product and returns it to manufacturing unit. The proposed study also considers hybrid processing facility, which is responsible for processing new and used product, simultaneously. Fig. 1 demonstrates the structure of the proposed study (Wu et al., 2006).

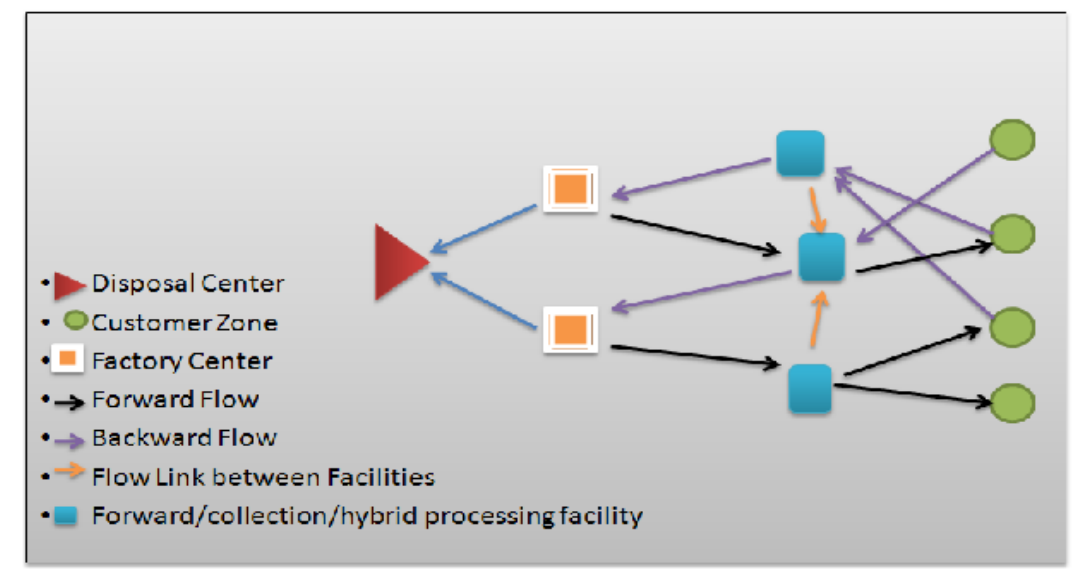

Fig. 1. The structure of the proposed study

The following notations are used for the proposed study of this paper.

J $\quad$ Fixed points for customers

$\mathrm{J}_{1} \quad$ Customers with forward demand

$\mathrm{J}_{2} \quad$ Customers with return demand

$i \quad$ Potential facilities for customers type $A$

$l \quad$ Fixed location for facility type $l$

$c f_{i}^{f} \quad$ Fixed cost associated with establishment of forward facility

$c f_{i}{ }^{h} \quad$ Fixed cost associated with establishment of hybrid facility

$c f_{i}{ }^{r} \quad$ Fixed cost associated with establishment of return facility

$q_{j}^{f} \quad$ Demand associated with establishment of forward facility

$q_{j}^{r} \quad$ The amount of returned product for customer $j$

$q_{i} \quad$ Quality of producer type $i$

$d_{i j} \quad$ Distance between customer $i$ and customer $j$ 


$\begin{array}{ll}\rho_{i j} & \text { Attractiveness of producer } i \text { for customer } j \\ b & \text { Buying power (Constant for all customers) } \\ d_{j b_{j}} & \text { Distance of customer type j from existing facilities } \\ S_{i}^{f} & \text { Capacity of forward distribution center for forward products } \\ S_{i}^{h r} & \text { Capacity of hybrid distribution center for return products } \\ S_{i}^{r} & \text { Capacity of collection center for return products } \\ S_{i}^{h f} & \text { Capacity of hybrid distribution center for forward products } \\ C_{l i} & \text { The cost of transportation of product type } l \text { to customer } i \\ C_{l i} & \text { Cost of recycling product } \\ p & \text { Price of returned good } \\ p_{l} & \text { Price of returned good sent by center } l \\ C_{i k}^{\prime} & \text { Cost of transportation between center } i \text { and } k \\ C_{l} & \text { Capacity of plant } l \text { for shipment } \\ \gamma & \text { Percentage of products returned } \\ J(B) & \text { Set of facilities associated with existing facilities whose locations are closer to } B\end{array}$

The following variables are considered for the proposed study of this paper.

$x_{i}^{f} \quad$ A binary variable, which is one if a forward facility is opened on place $i$, zero, otherwise

$x_{i}^{h} \quad$ A binary variable, which is one if a hybrid facility is opened on place $i$, zero, otherwise

$x_{i}^{r} \quad$ A binary variable, which is one if a return facility is opened on place $i$, zero, otherwise

$x_{i j} \quad$ A binary variable, which is one if customer $j$ selects producer $i$

$y_{k i} \quad$ The amount of new product sent from center $k$ to center $i$

$u_{l i} \quad$ The amount of new product sent from producer $l$ to center $i$

$u_{i l}^{\prime} \quad$ The amount of return product sent from producer $l$ to center $i$

$\rho_{i j} \quad$ The attractiveness of facility $i$ for customer $j$ defined as $\rho_{i j}=\frac{q_{i}}{\left(1+d_{i j}^{2}\right)}$

Based on the notations and variables defined, we now present the problem statement as follows,

$\max$ Share $_{A}=\sum_{i=1}^{m} \sum_{j}\left(b_{j} \rho_{i j} X_{i j}\right)$

subject to

$\sum_{i \in I} x_{i j} \leq 1 \quad \forall j \in J_{1} \cup J_{2}$

$x_{i j} \leq x_{i}^{f}+x_{i}^{h} \quad \forall i \in J(B), j \in J_{1}$

$x_{i j} \leq x_{i}^{h}+x_{i}^{r} \quad \forall i \in J(B), j \in J_{2}$

$x_{i}^{f}+x_{i}^{h}+x_{i}^{r} \leq 1 \quad \forall i \in I$

$\sum_{j \in J_{1}} q_{j}^{f} x_{i j}=u_{l i}+\sum_{k \in I} y_{k i} \quad \forall i \in I$

$\sum_{j \in J_{2}} q_{j}^{r} X_{i j} \leq S_{i}^{h r} x_{i}^{h}+S_{i}^{r} x_{i}^{r} \quad \forall i \in I$

$u_{l i}+\sum_{k \in I} y_{k i} \leq S_{i}^{h f} x_{i}^{h}+S_{i}^{f} x_{i}^{f} \quad \forall i \in I$ 


$$
\begin{aligned}
& \sum_{i \in I}^{338} u_{l i} \leq C_{l}^{L} \\
& y_{k i} \leq u_{l i}+\gamma \sum_{j \in J_{2}} q_{j}^{r} x_{i j} \quad \forall i, k \in I \\
& u_{i l}^{\prime} \leq \sum_{j \in J_{2}}(1-\gamma) q_{j}^{r} x_{i j} \quad \forall i \in I \\
& \left(c f_{i}^{f} x_{i}^{f}+c f_{i}^{h} x_{i}^{h}+c f_{i}^{r} x_{i}^{r}\right)+u_{l i} \cdot C_{l i}+\sum_{k \in I} y_{k i} \cdot C_{i k}^{\prime}+\sum_{j_{2}} p \cdot q_{j}^{r} x_{i j} \\
& \quad+\gamma \sum_{j} p^{R} \cdot q_{j}^{r} x_{i j} \leq \sum_{j, j_{2}}\left(\left(q_{j}^{\prime}+q_{j}^{r}\right) \cdot b_{j} x_{i j}\right)+(1-\gamma) \sum_{j} p \cdot q_{j}^{r} x_{i j} \forall i \\
& x_{i j}, x_{i}^{f}, x_{i}^{h}, x_{i}^{r} \in\{0,1\}, y_{k i}, u_{l i}, u_{i l}^{\prime} \geq 0 \quad \forall i, j, l
\end{aligned}
$$

The objective function maximizes the market share of producer type $A$ by considering the new rival. Eq. (1) specifies that each demand receive its service from only one producer. According to Eq. (2) and Eq. (3), any customer demanding forward and return product can only get its service from an open center, respectively. Eq. (5) assures that only one facility can be opened in each place. According to Eq. (6), Demand associated with establishment of forward facility should be equal to the amount of new product sent from center $k$ to center $i$ and the amount of new product sent from producer $l$ to center $i$. Eq. (7) specifies that sum of capacity of collection center for return products and capacity of hybrid distribution center for forward products must be at least equal to the amount of returned product for customer $j$. According to Eq. (8), capacity of forward distribution center for forward products and capacity of hybrid distribution center for forward products should at least equal to the amount of returned product for customer $j$. Eq. (9) indicates that the amount of new product sent from producer $l$ to center $i$ must be at least equal to capacity of plant $l$ for shipment. According to Eqs. (10-11), either the amount of new product sent from center $k$ to center $i$ must be less than equal to the amount of new product sent from producer $l$ to center $i$ plus the amount of returned product for customer $j$ or the amount of return product sent from producer $l$ to center $i$ should be the amount of returned product for customer $j$. Finally, Eq. (13) demonstrates the type of different variables used for the proposed study of this paper.

\section{Solution strategy}

The proposed study of this paper uses Lagrangian relaxation (Geoffrion \& Bride, 1978; Klincewicz, \& Luss, 1986) similar to the work by Wu et al. (2006) as follows,

$$
\max \operatorname{Share}_{A}=\sum_{i=1}^{m} \sum_{j}\left(b_{j} \rho_{i j} X_{i j}\right)-\sum_{j=1}^{n} \lambda_{j}\left(1-\sum_{i=1}^{m} X_{i j}\right)
$$

where the Lagrange multiplier in Eq. (14) is updated as follows,

$$
\lambda_{i+1}=\max \left(0, \lambda_{i}+t_{i} h_{i}\right) \text {. }
$$

In Eq. (15), $t_{i}$ is updated as follows,

$$
t_{i}=\frac{\varphi\left(Z_{u p}-\theta\left(\lambda^{*}\right)\right)}{\left\|h_{i}\right\|^{2}} .
$$

Next, we present details of our implementation for some problems using some direct and Lagrangian relaxation. For the performance measurement, the following input data are used. 


$$
\begin{aligned}
& \mathrm{cff} \sim U[0,90]+U[100,110] \sqrt{S_{i}^{f}} \\
& S_{i}^{h r} \sim U[5,100] \\
& \mathrm{cfh} \sim U[0,90]+U[100,110] \sqrt{S_{i}^{f h}+S_{i}^{r h}} \quad S_{i}^{r} \sim U[5,110] \\
& \operatorname{cfr} \sim U[0,90]+U[100,110] \sqrt{S_{i}^{r}} \quad p_{l} \sim U[6,9] \\
& S_{i}^{f} \sim U[10,160] \\
& q_{j}^{f} \sim U[5,35] \\
& S_{i}^{h f} \sim U[8,110] \\
& q_{j}^{r} \sim U[2,25] \\
& q_{i} \sim U[0.5,1]
\end{aligned}
$$

Table 1 shows details of our implementation for some randomly generated data for various centers $\times$ customers. In Table $1, Z_{M}, Z_{L R}$ represent the objective function for direct implementation versus Lagrangian relaxation, respectively. In addition, Relative Gap and Absolute Gap are measured as follows,

Relative Gap $=\frac{\left|Z_{L R}-Z_{M}\right|}{Z_{L R}}$
Absolute Gap $=\left|Z_{L R}-Z_{M}\right|$

Table 1

The summary of the proposed study for various centers and customers

\begin{tabular}{ccccc}
\hline Instance & $Z_{M}$ & $Z_{L R}$ & Relative Gap & Absolute Gap \\
\hline $10 \times 10$ & 10081.9763 & 10429.9378 & 347.9615 & 0.03336 \\
$10 \times 20$ & 13574.2884 & 13617.3473 & 43.0589 & 0.00316 \\
$10 \times 30$ & 13737.3347 & 13737.3347 & 0 & 0 \\
$40 \times 20$ & 18073.0894 & 18485.6557 & 412.5663 & 0.02231 \\
$50 \times 20$ & 27423.1351 & 30741.1081 & 3317.973 & 0.107933 \\
$80 \times 20$ & 29436.0866 & 30764.8547 & 1328.77 & 0.04319 \\
$100 \times 20$ & 29512.0075 & 30751.716565 & 1002.803 & 0.03261 \\
$100 \times 30$ & 44309.8313 & 44619.013895 & 309.1825 & 0.0069 \\
$100 \times 50$ & 83672.6036 & 83698.848054 & 26.24545 & 0.00031 \\
$100 \times 100$ & 175589.709626 & 175652.452551 & 62.74293 & 0.00035 \\
$120 \times 20$ & 30172.2207 & 32779.0838 & 2606.86 & 0.07953 \\
$200 \times 20$ & 30457.8298 & 30930.429917 & 472.6 & 0.01528 \\
$30 \times 30$ & 15412.2881 & 15412.3325 & 0.0444 & 0 \\
$50 \times 30$ & 39230.3864 & 40347.9777 & 1117.59 & 0.0277 \\
$100 \times 30$ & 46127.5311 & 46599.785511 & 472.254 & 0.01013 \\
$20 \times 40$ & 19315.1351 & 19316.25855 & 1.1229 & 0 \\
$30 \times 40$ & 27147.7267 & 27152.1485 & 4.4218 & 0.00016 \\
$40 \times 40$ & 34900.6338 & 34907.2737 & 6.6399 & 0.00019 \\
$80 \times 40$ & 56343.6868 & 56355.5940 & 11.908 & 0.00021 \\
$200 \times 50$ & 89616.9761 & 89639.4695 & 22.4929 & 0.00025 \\
$200 \times 100$ & 187404.134342 & 187464.5263 & 60.392 & 0.00032 \\
$200 \times 200$ & 379959.9864 & 383016.362096 & 3056.38 & 0.00798 \\
\hline $700 \times 400$ & - & 781863.7428 & - & - \\
\hline $700 \times 500$ & - & 977310.8823 & - & - \\
\hline
\end{tabular}

As we can observe from the results of Table 1, the optimal solutions of the direct method is the same as the Lagranigan relaxation for small instances. However, as the size of the problem increases the gap between two methods increases. Fig. 2 demonstrates the results for 10 customers and the results indicate that when the number of customers increases from 10 to 30 , the relative gap decreases. 


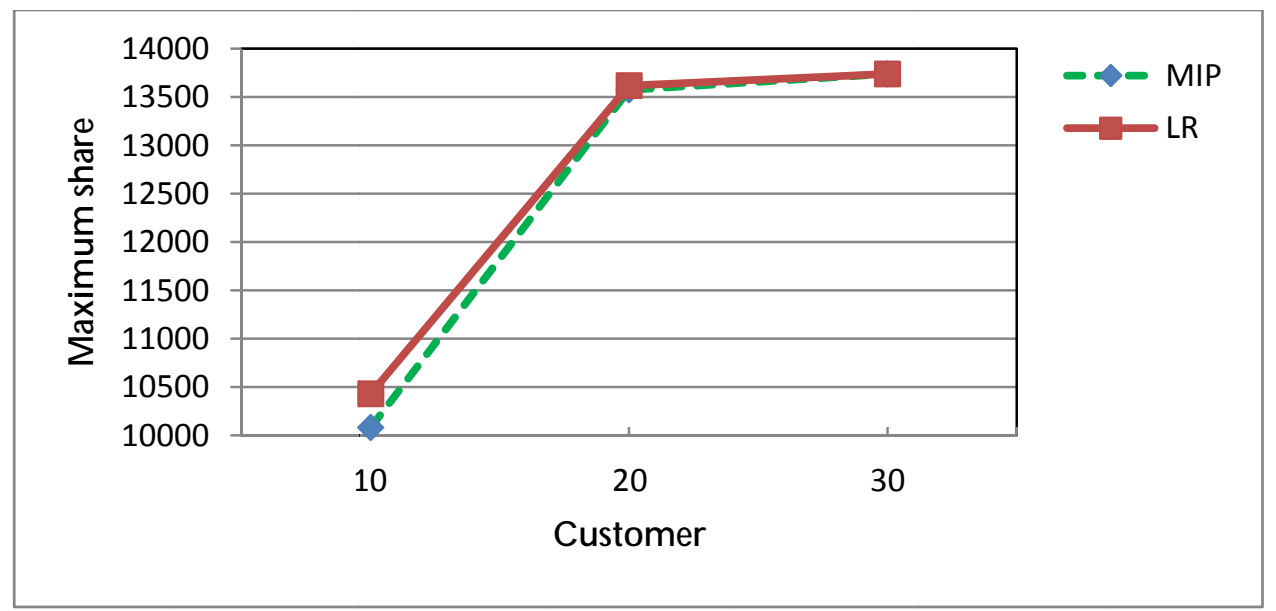

Fig. 2. The summary of the performance of the proposed study for 10-30 customers

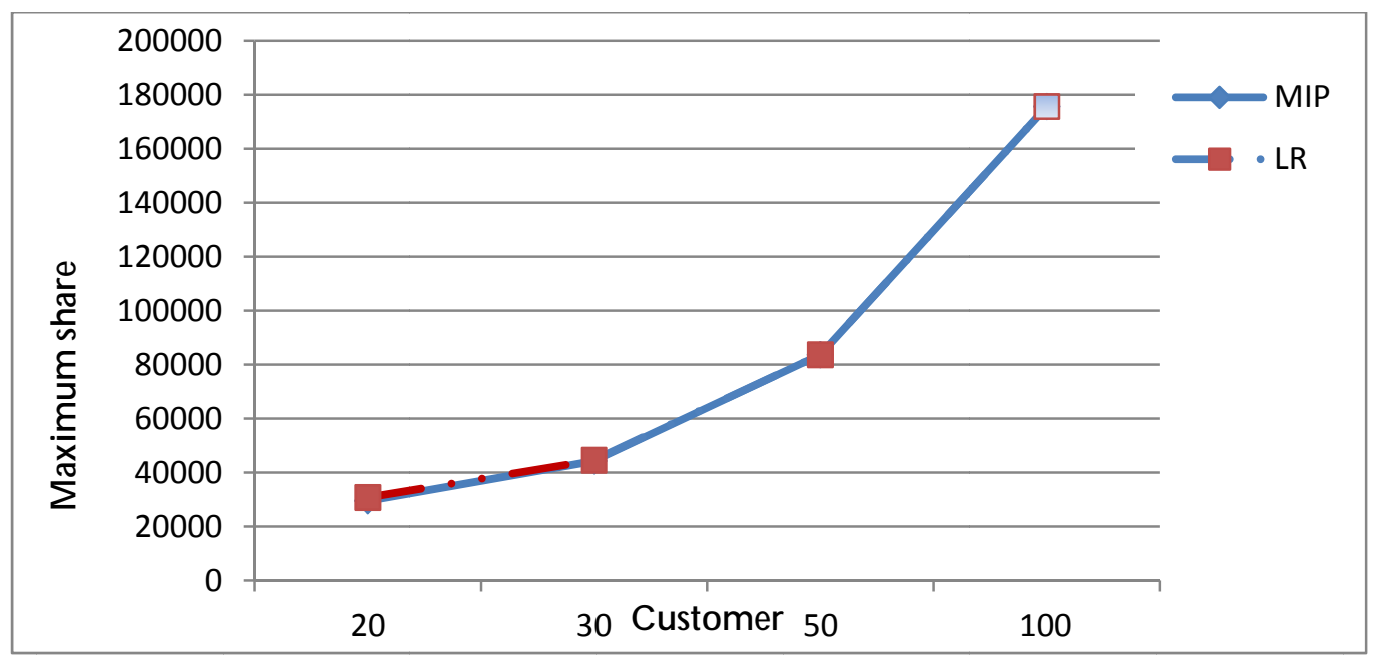

Fig. 3. The summary of the proposed study for 20-100 customers

According to Fig. 3, as the number of customer increases to 100, there is not any significant difference between two methods. Finally, Fig. 4 shows details of the relative gap between two methods.

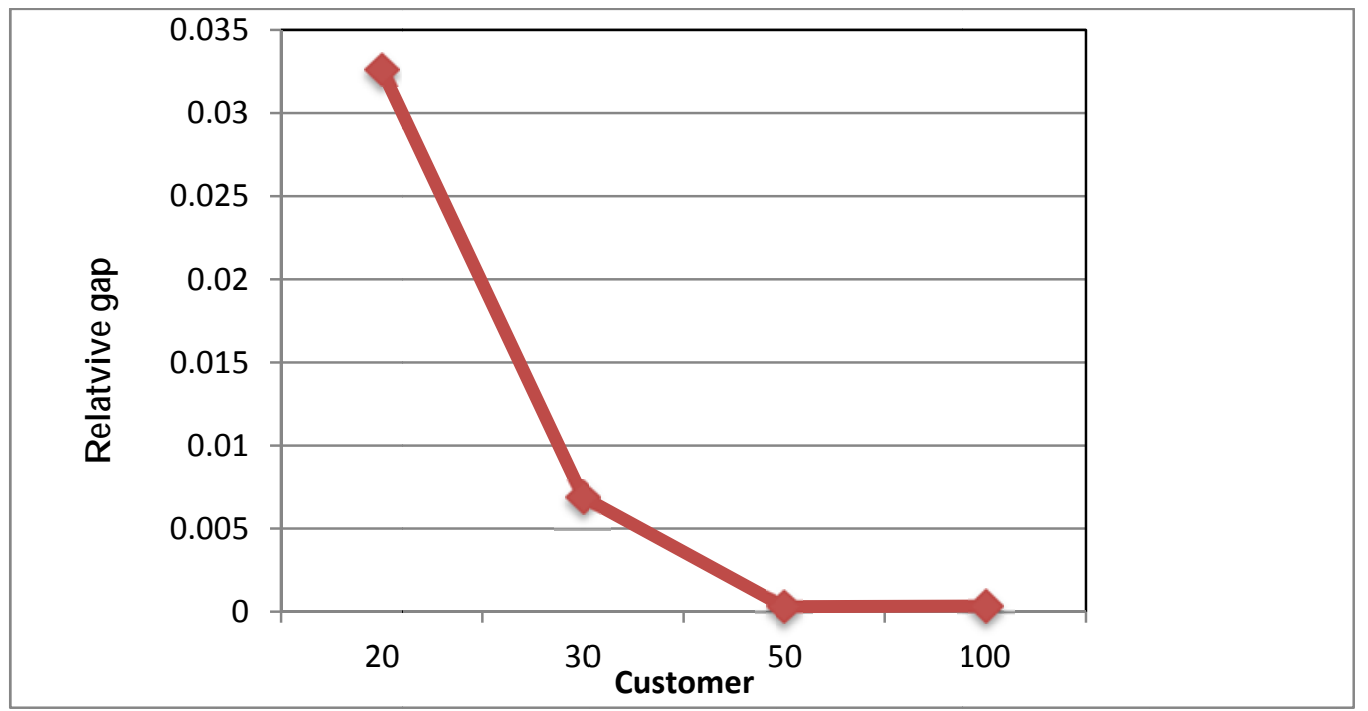

Fig. 4. The results of relative gap 


\section{Conclusion}

In this paper, we have presented a facility location strategy where there is one product for distribution. The proposed study has assumed that there are some potential competitors coming to market. The study has also considered that part of products are returned to manufacturer for re-sell programs. The proposed study has implemented Lagrangian relaxation to compare the performance of the proposed studies. The results have indicated that both methods were capable of providing some optimal solutions for small instances. However, as the size of the problem increases, only Lagrangian relaxation was able to solve the problem for real-world case studies. The study of this paper can be considered for problems with more realistic assumptions. For instance, Ozgen and Gulsun (2014) combined possibilistic linear programming and fuzzy AHP for solving the multi-objective capacitated multi-facility location problem. The proposed study of this paper can incorporate the Lagrangian relaxation to solve multi-objective type of problems. Aydin and Murat (2013) applied swarm intelligence based sample average approximation algorithm for the capacitated reliable facility location problem and we may use other meth-heuristics to solve the proposed study of this paper and we leave for interested researchers for future studies.

\section{Acknowledgement}

The authors would like to thank the anonymous referees for constructive comments on earlier version of this paper.

\section{References}

Avella, P., Boccia, M., Sforza, A., \& Vasil'ev, I. (2009). An effective heuristic for large-scale capacitated facility location problems. Journal of Heuristics, 15(6), 597-615.

Aydin, N., \& Murat, A. (2013). A swarm intelligence based sample average approximation algorithm for the capacitated reliable facility location problem. International Journal of Production Economics, 145(1), 173-183

Geoffrion, A., \& Bride, R. M. (1978). Lagrangean relaxation applied to capacitated facility location problems. AIIE transactions, 10(1), 40-47.

Klincewicz, J. G., \& Luss, H. (1986). A Lagrangian relaxation heuristic for capacitated facility location with single-source constraints. Journal of the Operational Research Society, 37(5), 495500.

Küçükdeniz, T., Baray, A., Ecerkale, K., \& Esnaf, Ş. (2012). Integrated use of fuzzy c-means and convex programming for capacitated multi-facility location problem. Expert Systems with Applications, 39(4), 4306-4314

Jain, K., \& Vazirani, V. V. (2001). Approximation algorithms for metric facility location and kmedian problems using the primal-dual schema and Lagrangian relaxation. Journal of the ACM (JACM), 48(2), 274-296.

Manzour-al-Ajdad, S.M.H., Torabi, S.A., \& Eshghi, K. (2012). Single-Source Capacitated MultiFacility Weber Problem-An iterative two phase heuristic algorithm. Computers \& Operations Research, 39(7), 1465-1476.

Nauss, R. M. (1978). An improved algorithm for the capacitated facility location problem. Journal of the Operational Research Society, 29(12), 1195-1201.

Ozgen, D., \& Gulsun, B. (2014). Combining possibilistic linear programming and fuzzy AHP for solving the multi-objective capacitated multi-facility location problem. Information Sciences, 268(1), 185-201.

Pirkul, H., \& Jayaraman, V. (1998). A multi-commodity, multi-plant, capacitated facility location problem: formulation and efficient heuristic solution. Computers \& Operations Research, 25(10), 869-878.

Rahmaniani, R., \& Ghaderi, A. (2013). A combined facility location and network design problem with multi-type of capacitated links. Applied Mathematical Modelling, 37(9), 6400-6414 
Sridharan, R. (1995). The capacitated plant location problem. European Journal of Operational Research, 87(2), 203-213.

Yang, Z., Chu, F., \& Chen, H. (2012). A cut-and-solve based algorithm for the single-source capacitated facility location problem. European Journal of Operational Research, 221(3), 521532.

Wu, L. Y., Zhang, X. S., \& Zhang, J. L. (2006). Capacitated facility location problem with general setup cost. Computers \& Operations Research, 33(5), 1226-1241. 